\title{
Moderate Range Static Magnetic Field Promoted Variation of Blood Parameters: An In vitro Study
}

\author{
Bestoon T. Mustafa, Sardar P. Yaba and Asaad H. Ismail \\ Department of Physics, College of Education, Salahaddin University, \\ Erbil 44001, Kurdistan Region - F.R. Iraq
}

\begin{abstract}
This study was undertaken to investigate the influence of a homogenous and uniform static magnetic field (SMF) on the main blood cell counts in vitro experiment. Fresh blood samples were collected from albino rats and exposed to SMF $(2.4,6,25,50$, 75 , and $100 \mathrm{mT}$ ) versus $15-60 \mathrm{~min}$. Results showed a significant change of blood counts under the low field effects. A $2.4 \mathrm{mT}$ was a trend of white blood cells (WBCs) count increase non-linearly. However, a $6 \mathrm{mT}$ exposure reduced WBCs with about 39\%. Other variations fluctuated within $30 \%$. The $25 \mathrm{mT}$ decreased red blood cells (RBCs), hemoglobin, and hematocrit levels with $13 \%$ similarly. The lower exposure field, (2.4 and 6) $\mathrm{mT}$, and effects on RBCs were $6 \%$ fluctuation. The $6 \mathrm{mT}$ reduced platelet counts with half in comparison to control blood samples. About $20 \%$ increase obtained due to $50 \mathrm{mT}$ exposure during all period. None of 75 and $100 \mathrm{mT}$ exposures dominated blood counts alterations. The quiet magnetic field exposure for a certain time can be interesting to control blood cell count-related diseases.
\end{abstract}

Index Terms-Aggregation, Blood counts, Hematology, Optical microscopy, Static magnetic field.

\section{INTRODUCTION}

The effect of static magnetic field (SMF) on biological system has being concerned biomedical researches. The produced magnetic field from magnetic resonance imaging, transmission line, electrical appliance, and other electronic devices provided a tremendous study opportunity to evaluate the cellular effect of magnetic field (Chadwick and Lowesf, 1998; Okano and Ohkubo, 2005; Van Deventer, et al., 2005). Experimental results suggested several cellular disorders. Yet, the results were contradictory rather than declare a clear answer (Mustafa, et al., 2019; Vergallo and Dini, 2018). Main blood cells, white blood cells (WBCs), red blood cells (RBCs), and platelets (PLTs), are body defensive, oxygen support, and hemostasis precursors in animals (Ismail, 2015; Laith, et al., 2020). Several in vivo experiments were

\section{ARO-The Scientific Journal of Koya University}

Vol. VIII, No.1 (2020), Article ID: ARO.10612, 9 pages

DOI:10.14500/aro.10612

Received 20 December 2019; Accepted 18 April 2020

Regular research paper: Published 10 May 2020

Corresponding author's e-mail: bestoon.mustafa@su.edu.krd

Copyright (C) 2020 Bestoon T. Mustafa, Sardar P. Yaba and

Asaad H. Ismail. This is an open-access article distributed under the

Creative Commons Attribution License. conducted to reveal the influence of pulsed and SMF on blood cells. Nevertheless, only a few in vitro experiments explained the response of outcomes.

SMF is a time-independent field generated through passing a DC current through a coil (Mustafa, et al., 2020a). SMF can penetrate tissues and thermalize the molecules stabilization (Hashish, et al., 2008). According to the World Health Organization (World Health Organization, 2006), magnetic material found in some organic tissues and hence magnetic field can interact directly with several proteins, ions, and other cellular parts of animals.

RBCs are about $45 \%$ of blood content mainly cause blood viscosity manipulation. In addition, disorder in counts and morphology can cause pathological diseases or anima. Wister rats were exposed to SMF in vivo experiment (Chater, et al., 2006). A 13-day exposure, $128 \mathrm{mT}: 1 \mathrm{~h} /$ day, increased hematocrit percentage $(\mathrm{HTC} \%)$ and hemoglobin (HGB) contents with $7 \%$ and $12 \%$, respectively. Milovanovich, et al., 2016, examined 5 days: $1 \mathrm{~h} /$ day exposure effect of SMF on mice. The field was applied in two different directions: Up and down. A $128 \mathrm{mT}$ reduced WBCs counts and increased PLTs and HGB contents. Aida, et al., 2014, repeated the same experiment using a homogeneous SMF. Five days exposure rats resulted in an increase of HGB and WBCs counts: 10\% and $17 \%$, respectively. The interesting part of the correlated experiments showed an increase of blood WBCs and PLTs.

The influence of long period subchronic uniform SMF studied by Djordjevich, et al., 2012. Male Swiss-Webster mice exposed with $16 \mathrm{mT}$ : 28 days, lymphocytes changed significantly. A magnetic field with as low as $1.4 \mathrm{mT}$ : 28 days continuous exposure decreased PLTs and lymphocytes percentage significantly (Hashish, et al., 2008). Despite to conduct several in vivo researches to study the magnetic field effect on blood, there is a significant gap left to determine in vitro exposure effect on blood hematology. In this study, we investigated the effect of moderate $\operatorname{SMF}(2.4,6,25,50$, 75 , and $100 \mathrm{mT}$ ) on rats' blood counts.

\section{Materials AND MethodS}

\section{A. Housing Animals and Blood Collection}

In this experiment, 24 albino rats ages 5 months, weight between $230 \pm 20 \mathrm{~g}$, were participated in six selective groups 
(A, B, C, D, E, and F). Each group was with four rats kept in a standard plastic cage. The animals were kept in animal house long prior the experiment started date. They were suicide and fresh blood samples were collected. A standard

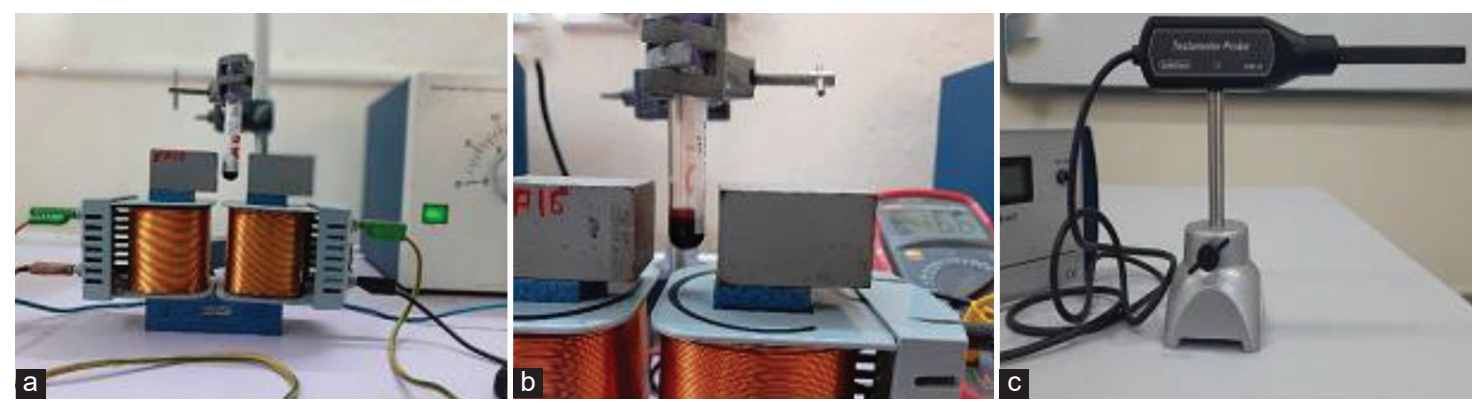

Fig. 1. (a-c) In vitro blood exposure system equipped with the digital Teslameter.
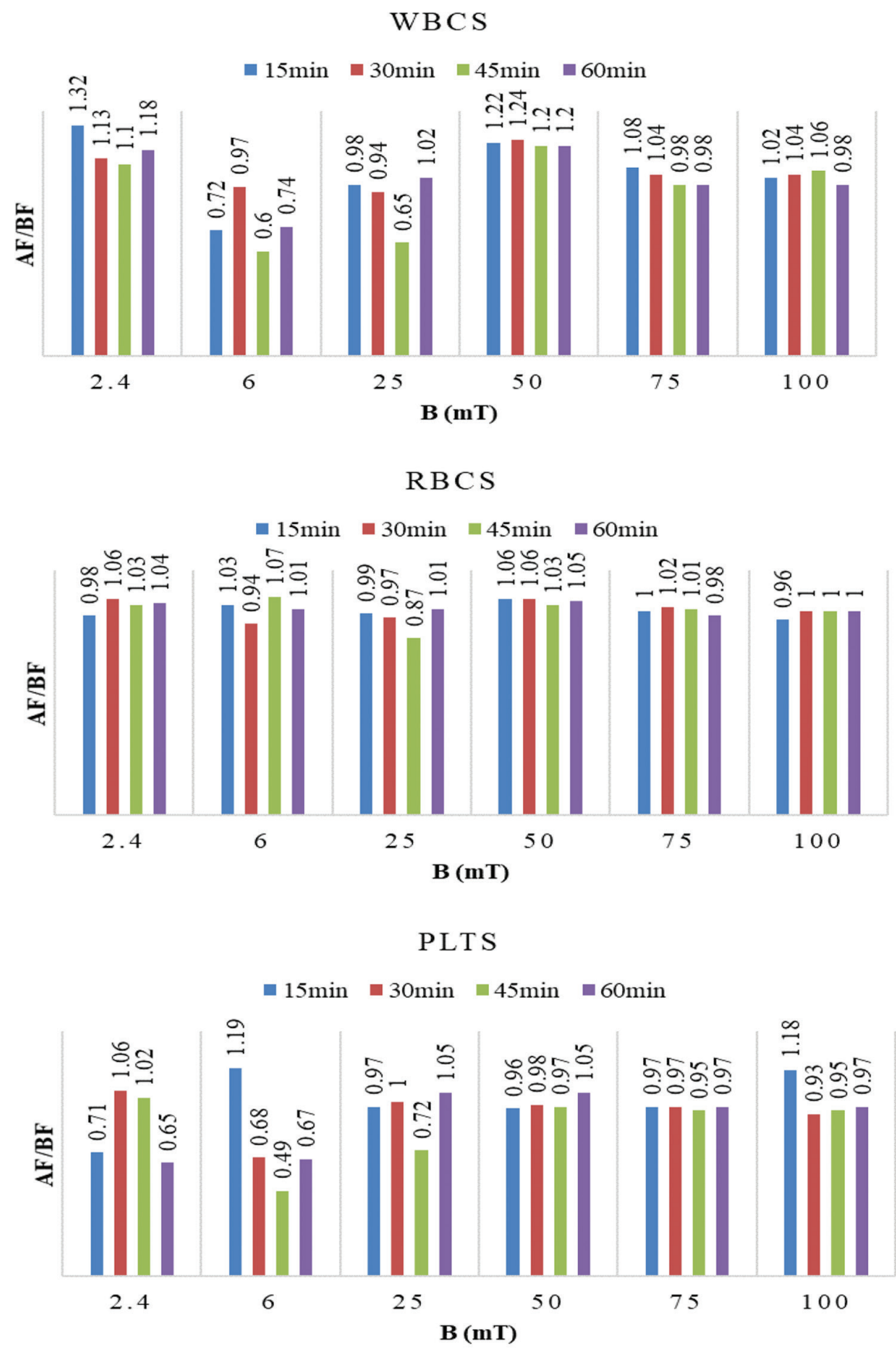

Fig. 2. Blood cells alteration based on exposure time and magnetic field intensity of white blood cells, red blood cells, and platelets. Y-axis represents the ratio between results after exposure to before exposure. A value above "1" represents an increase and below "1" is a decrease compared to control results. 
animal house was used in the Biology Department, College of Education, Salahaddin University, Erbil; living atmosphere was $12: 12 \mathrm{~h}$ light and darkness, photoperiod of $22 \pm 2^{\circ} \mathrm{C}$, received a standard rat chow and drink (tap water).

A mixture of xylazine and ketamine hydrochloride were prepared and used to anesthetize rats. Blood samples were withdrawn from cardiac puncture into $6 \mathrm{ml}$ tubes inner wall covered with Ethylenediaminetetraacetic acid anticoagulation. Collected blood from each rat were saved into containers and taken to exposure directly. Each of the groups was prepared for a certain exposure field $(2.4,6,25,50,75$, and $100 \mathrm{mT})$. The exposure time was

TABLE I

Blood Counts Under 2.4 Mt Static Magnetic Field Versus Exposure Period. A Few Changes are Significant $(P<0.05)$

\begin{tabular}{|c|c|c|c|c|c|}
\hline \multirow{2}{*}{$\begin{array}{l}\mathrm{B}=2.4 \mathrm{mT} \\
\text { Parameter/period }\end{array}$} & \multicolumn{5}{|c|}{ Exposure time } \\
\hline & Control & $15 \mathrm{~min}$ & $30 \mathrm{~min}$ & $45 \mathrm{~min}$ & $45 \mathrm{~min}$ \\
\hline WBCs $\left(10^{9} / 1\right)$ & $6 \pm 0.12$ & $7.9 \pm 0.38$ & $6.8 \pm 0.19$ & $6.6 \pm 0.33$ & $7.1 \pm 0.98$ \\
\hline $\operatorname{LYM}\left(10^{9} / 1\right)$ & $3.4 \pm 0.02$ & $3 \pm 0.25$ & $3.6 \pm 0.15$ & $3.5 \pm 0.04$ & $5.8 \pm 0.31$ \\
\hline LYM\% & $55.2 \pm 1.40$ & $38.8 \pm 3.11$ & $52 \pm 0.73$ & $52.4 \pm 1.11$ & $80.7 \pm 3.70$ \\
\hline $\operatorname{MID}\left(10^{9} / 1\right)$ & $0.7 \pm 0.02$ & $1 \pm 0.05$ & $0.4 \pm 0.09$ & $0.4 \pm 0.03$ & $0.6 \pm 0.001$ \\
\hline MID \% & $12.3 \pm 1.01$ & $13.4 \pm 0.16$ & $7 \pm 1.00$ & $6.3 \pm 0.90$ & $8.5 \pm 0.89$ \\
\hline $\operatorname{RBCs}\left(10^{12} / 1\right)$ & $6.69 \pm 1.40$ & $6.53 \pm 0.74$ & $7.06 \pm 0.39$ & $6.88 \pm 1.03$ & $6.95 \pm 0.52$ \\
\hline $\operatorname{HGB}(\mathrm{g} / \mathrm{dl})$ & $11.8 \pm 0.49$ & $12.5 \pm 2.10$ & $12.9 \pm 1.78$ & $12.2 \pm 0.88$ & $13.7 \pm 2.55$ \\
\hline НCT\% & $32.1 \pm 3.21$ & $33.8 \pm 3.09$ & $34 \pm 1.84$ & $33.1 \pm 0.98$ & $39.1 \pm 2.46$ \\
\hline $\operatorname{MCV}(\mathrm{fl})$ & $48 \pm 3.21$ & $51.8 \pm 2.76$ & $48.1 \pm 4.01$ & $48 \pm 3.29$ & $56.2 \pm 2.10$ \\
\hline $\mathrm{MCH}(\mathrm{pg})$ & $17.7 \pm 1.02$ & $19.2 \pm 3.03$ & $18.2 \pm 0.65$ & $17.7 \pm 0.93$ & $19.8 \pm 1.40$ \\
\hline $\mathrm{MCHC}(\mathrm{g} / \mathrm{dl})$ & $36.9 \pm 2.09$ & $37.1 \pm 3.54$ & $37.9 \pm 2.94$ & $36.9 \pm 3.65$ & $35.2 \pm 2.93$ \\
\hline PDWa (fl) & $9.3 \pm 1.21$ & 9.11 .09 & $9.2 \pm 0.94$ & $9.4 \pm 1.43$ & $9.5 \pm 0.43$ \\
\hline PDW\% & $38 \pm 2.13$ & $38.8 \pm 1.54$ & $37.5 \pm 4.22$ & $38 \pm 3.23$ & $39.6 \pm 1.84$ \\
\hline РCT\% & $0.44 \pm 0.00$ & $0.3 \pm 0.01$ & $0.46 \pm 0.09$ & $0.45 \pm 0.04$ & $0.29 \pm 0.08$ \\
\hline P-LCR \% & $5 \pm 1.03$ & $4.9 \pm 0.94$ & $3.7 \pm 0.84$ & $4.6 \pm 0.53$ & $6.9 \pm 0.31$ \\
\hline P-LCC $\left(10^{9} / 1\right)$ & $35 \pm 1.50$ & $24 \pm 1.01$ & $27 \pm 2.09$ & $32 \pm 2.43$ & $31 \pm 2.04$ \\
\hline
\end{tabular}

WBCs: White blood cells, RBCs: Red blood cells, HGB: Hemoglobin, PLT: Platelet

TABLE II

Blood Counts Under 6 Mt Static Magnetic Field Versus Exposure Period. A Few Changes are Significant $(P<0.05)$

\begin{tabular}{|c|c|c|c|c|c|}
\hline \multirow{2}{*}{$\frac{\mathrm{B}=6 \mathrm{mT}}{\text { Parameter/period }}$} & \multicolumn{5}{|c|}{ Exposure time } \\
\hline & Control & $15 \mathrm{~min}$ & $30 \mathrm{~min}$ & $45 \mathrm{~min}$ & $45 \mathrm{~min}$ \\
\hline WBCs $\left(10^{9} / 1\right)$ & $8.8 \pm 0.38$ & $6.3 \pm 0.24$ & $8.5 \pm 0.47$ & $5.3 \pm 0.14$ & $6.5 \pm 0.62$ \\
\hline $\operatorname{LYM}\left(10^{9} / 1\right)$ & $3.6 \pm 0.09$ & $3.3 \pm 0.33$ & $4.6 \pm 0.19$ & $2.3 \pm 0.05$ & $2.6 \pm 0.19$ \\
\hline LYM\% & $41.8 \pm 0.38$ & $52.2 \pm 0.38$ & $53.9 \pm 0.52$ & $40.2 \pm 0.24$ & $40 \pm 0.94$ \\
\hline $\operatorname{MID}\left(10^{9} / 1\right)$ & $0.5 \pm 0.05$ & $0.4 \pm 0.02$ & $0.4 \pm 0.02$ & $0.25 \pm 0.02$ & $0.4 \pm 0.03$ \\
\hline MID \% & $6.1 \pm 0.14$ & $6.4 \pm 0.33$ & $5.2 \pm 0.00$ & $7.5 .4 \pm 0.24$ & $6.1 \pm 0.38$ \\
\hline $\operatorname{RBCs}\left(10^{12} / 1\right)$ & $6.64 \pm 0.16$ & $6.85 \pm 0.12$ & $6.26 \pm 0.35$ & $7.09 \pm 0.28$ & $6.69 \pm 0.15$ \\
\hline HGB (g/dl) & $12.5 \pm 0.24$ & $12.2 \pm 0.09$ & $12.2 \pm 0.09$ & $14.2 \pm 0.42$ & $12.9 \pm 0.52$ \\
\hline HCT (\%) & $34.4 \pm 0.33$ & $33.1 \pm 0.33$ & $32.6 \pm 0.19$ & $38.1 \pm 0.38$ & $35 \pm 0.94$ \\
\hline MCV (fl) & $51.8 \pm 0.24$ & $48.4 \pm 0.61$ & $52 \pm 0.14$ & $52.4 \pm 0.28$ & $52.30 \pm 0.8$ \\
\hline MCH (pg) & $18.9 \pm 0.14$ & $17.8 \pm 0.28$ & $19.4 \pm 0.28$ & $20.8 \pm 0.71$ & $19.2 \pm 0.57$ \\
\hline MCHC (g/dl) & $36.5 \pm 0.24$ & $36.7 \pm 0.24$ & $37.4 \pm 0.66$ & $35.3 \pm 0.61$ & $36.8 \pm 1.79$ \\
\hline PDWa (fl) & $9.3 \pm 0.14$ & $9.4 \pm 0.19$ & $9 \pm 0.24$ & $8.3 \pm 0.38$ & $9.2 \pm 0.24$ \\
\hline PDW\% & $39.2 \pm 0.75$ & $38 \pm 0.24$ & $38 \pm 0.47$ & $39.2 \pm 0.71$ & $39.4 \pm 00.75$ \\
\hline РCT\% & $0.34 \pm 0.03$ & $0.42 \pm 0.04$ & $0.23 \pm 0.01$ & $0.42 \pm 0.02$ & $0.22 \pm 0.03$ \\
\hline P-LCR $\%$ & $5.3 \pm 0.14$ & $4.5 \pm 0.14$ & $4.7 \pm 0.14$ & $4.5 \pm 0.09$ & $5 \pm 0.24$ \\
\hline P-LCC $\left(10^{9} / 1\right)$ & $29 \pm 1.41$ & $29 \pm 1.04$ & $17 \pm 0.47$ & $12 \pm 1.47$ & $18 \pm 00.47$ \\
\hline
\end{tabular}

WBCs: White blood cells, RBCs: Red blood cells, HGB: Hemoglobin, PLT: Platelet 
examined versus magnetic field intensity. Controls blood samples were prepared from the same collected blood of each rat. For example, a $6 \mathrm{ml}$ blood collected from each rat divided into 5 tubes, one control and four taken to exposure with the same magnetic field versus exposure time $(15,20,45$, and $60 \mathrm{~min})$. The same procedure repeated for all rats in each group and then average was taken.

TABLE III

Blood Counts Under 25 Mt Static Magnetic Field Versus Exposure Period. A Few Changes are Significant $(P<0.05)$

\begin{tabular}{|c|c|c|c|c|c|}
\hline \multirow{2}{*}{$\begin{array}{l}\mathrm{B}=25 \mathrm{mT} \\
\text { Parameters/period }\end{array}$} & \multicolumn{5}{|c|}{ Exposure time } \\
\hline & Control & $15 \mathrm{~min}$ & $30 \mathrm{~min}$ & $45 \mathrm{~min}$ & $60 \mathrm{~min}$ \\
\hline WBCs $\left(10^{9} / 1\right)$ & $8.6 \pm 0.19$ & $8.4 \pm 0.66$ & $8.1 \pm 0.42$ & $5.6 \pm 0.28$ & $8.8 \pm 0.17$ \\
\hline LYM $\left(10^{9} / 1\right)$ & $5.8 \pm 0.52$ & $5.9 \pm 0.42$ & $5.8 \pm 0.33$ & $3.9 \pm 0.38$ & $6.1 \pm 0.61$ \\
\hline $\operatorname{MID}\left(10^{9} / 1\right)$ & $0.5 \pm 0.05$ & $0.8 \pm 0.05$ & $0.5 \pm 0.09$ & $0.8 \pm 0.05$ & $0.6 \pm 0.09$ \\
\hline MID $\%$ & $6.8 \pm 0.09$ & $9.4 \pm 0.38$ & $7 \pm 0.71$ & $14.1 \pm 1.13$ & $6.7 \pm 0.38$ \\
\hline $\operatorname{RBCs}\left(10^{12} / 1\right)$ & $6.65 \pm 0.25$ & $6.59 \pm 0.57$ & $6.48 \pm 0.48$ & $5.77 \pm 0.30$ & $6.74 \pm 0.36$ \\
\hline HGB (g/dl) & $12.4 \pm 0.19$ & $12.3 \pm 0.33$ & $12.1 \pm 0.66$ & $10.8 \pm 0.47$ & $12.5 \pm 0.94$ \\
\hline НСТ $\%$ & $33.5 \pm 0.71$ & $33 \pm 0.94$ & $32.4 \pm 0.61$ & $29 \pm 1.13$ & $33.9 \pm 0.71$ \\
\hline MCV (fl) & $50.4 \pm 0.75$ & $50 \pm 1.18$ & $50 \pm 1.18$ & $50.2 \pm 1.08$ & $50.2 \pm 1.08$ \\
\hline $\mathrm{MCH}(\mathrm{pg})$ & $18.6 \pm 0.19$ & $18.7 \pm 0.61$ & $18.7 \pm 0.85$ & $18.7 \pm 0.42$ & $18.6 \pm 0.52$ \\
\hline $\operatorname{MCHC}(\mathrm{g} / \mathrm{dl})$ & $36.9 \pm 0.90$ & $37.3 \pm 0.80$ & $37.4 \pm 0.99$ & $37.3 \pm 1.13$ & $37.1 \pm 0.71$ \\
\hline PDWa (fl) & $8.8 \pm 0.33$ & $8.6 \pm 0.28$ & $8.7 \pm 0.52$ & $9.6 \pm 0.80$ & $8.7 \pm 0.42$ \\
\hline PDW\% & $38.4 \pm 1.27$ & $37.8 \pm 1.32$ & $37.7 \pm 1.27$ & $39.4 \pm 0.38$ & $38 \pm 0.75$ \\
\hline РCТ\% $\%$ & $0.35 \pm 0.07$ & $0.34 \pm 0.04$ & $0.35 \pm 0.05$ & $0.28 \pm 0.03$ & $0.37 \pm 0.06$ \\
\hline P-LCR\% & $3.3 \pm 0.33$ & $2.8 \pm 0.09$ & $2.7 \pm 0.14$ & $4.5 \pm 0.09$ & $2.70 \pm 0.33$ \\
\hline P-LCC $\left(10^{9} / 1\right)$ & $20 \pm 1.25$ & $16 \pm 0.75$ & $16 \pm 0.19$ & $20 \pm 1.70$ & $17 \pm 0.80$ \\
\hline
\end{tabular}

WBCs: White blood cells, RBCs: Red blood cells, HGB: Hemoglobin, PLT: Platelet

TABLE IV

Blood Counts Under 50 Mt of Static Magnetic Field Versus Exposure Period. A Few Changes are Significant $(P<0.05)$

\begin{tabular}{|c|c|c|c|c|c|}
\hline \multirow{2}{*}{$\begin{array}{l}\mathrm{B}=50 \mathrm{mT} \\
\text { Parameters/period }\end{array}$} & \multicolumn{5}{|c|}{ Exposure time } \\
\hline & Control & $15 \mathrm{~min}$ & $30 \mathrm{~min}$ & $45 \mathrm{~min}$ & $60 \mathrm{~min}$ \\
\hline WBCs $\left(10^{9} / 1\right)$ & $5.4 \pm 0.38$ & $6.6 \pm 0.41$ & $6.7 \pm 0.61$ & $6.5 \pm 0.37$ & $6.5 \pm 0.14$ \\
\hline $\operatorname{LYM}\left(10^{9} / 1\right)$ & $3.8 \pm 0.09$ & $4.7 \pm 0.14$ & $4.6 \pm 0.37$ & $4.7 \pm 0.14$ & $4.7 .7 \pm 0.35$ \\
\hline LYM\% & $68.4 \pm 1.60$ & $70.2 \pm 0.85$ & $68.5 \pm 0.94$ & $72 \pm 0.47$ & $72 \pm 0.75$ \\
\hline $\operatorname{MID}\left(10^{9} / 1\right)$ & $0.3 \pm 0.02$ & $0.4 \pm 0.05$ & $0.4 \pm 0.05$ & $0.4 \pm 0.05$ & $0.4 .4 \pm 0.09$ \\
\hline MID $\%$ & $6.2 \pm 0.38$ & $6.4 \pm 0.28$ & $6.3 \pm 0.57$ & $6.5 \pm 0.71$ & $6.5 .5 \pm 0.38$ \\
\hline $\operatorname{RBCs}\left(10^{12} / 1\right)$ & $6.21 \pm 0.37$ & $6.59 \pm 0.19$ & $6.59 \pm 0.33$ & $6.41 \pm 0.56$ & $6.41 \pm 0.47$ \\
\hline HGB (g/dl) & $12.6 \pm 0.75$ & $13.5 \pm 0.24$ & $13.6 \pm 0.75$ & $13.2 \pm 0.61$ & $13.2 \pm 0.94$ \\
\hline НCТ $\%$ & $37.1 \pm 0.52$ & $38.8 \pm 1.32$ & $39 \pm 0.24$ & $38.3 \pm 0.57$ & $38.3 \pm 0.85$ \\
\hline MCV (fl) & $59.7 \pm 0.33$ & $58.8 \pm 1.32$ & $59.5 \pm 0.94$ & $59.7 \pm 0.33$ & $59.7 \pm 0.37$ \\
\hline $\mathrm{MCH}(\mathrm{pg})$ & $20.3 \pm 0.33$ & $20.6 \pm 0.75$ & $20.8 \pm 0.75$ & $20.7 \pm 0.28$ & $20.7 \pm 0.99$ \\
\hline $\operatorname{MCHC}(\mathrm{g} / \mathrm{dl})$ & $34 \pm 0.61$ & $34.9 \pm 0.90$ & $34.9 \pm 0.52$ & $34.6 \pm 0.47$ & $34.6 \pm 0.47$ \\
\hline PDWa (fl) & $9.7 \pm 0.33$ & $8.7 \pm 0.14$ & $8.8 \pm 0.33$ & $8.9 \pm 0.42$ & $8.9 \pm 0.71$ \\
\hline PDW\% & $41.1 \pm 0.42$ & $38.4 \pm 0.94$ & $38.2 \pm 0.66$ & $38.9 \pm 0.90$ & $38.9 \pm 0.80$ \\
\hline РCT\% & $0.32 \pm 0.01$ & $0.27 \pm 0.82$ & $0.29 \pm 0.03$ & $0.28 \pm 0.05$ & $0.28 \pm 0.07$ \\
\hline P-LCR \% & $8.90 \pm 0.42$ & $3.70 \pm 0.14$ & $4.30 \pm 0.38$ & $4.20 \pm 0.52$ & $4.2 \pm 0.66$ \\
\hline P-LCC $\left(10^{9} / 1\right)$ & $44 \pm 0.94$ & $27 \pm 1.23$ & $21 \pm 0.71$ & $20 \pm 1.60$ & $20 \pm 1.65$ \\
\hline
\end{tabular}

WBCs: White blood cells, RBCs: Red blood cells, HGB: Hemoglobin, PLT: Platelet 


\section{B. Exposure Method}

Solenoid coils were used to generate a highly stable and uniform SMF (Fig. 1). Two coils were connected and the magnetic field was measured between the iron cores using a Teslameter. A magnetic field with intensity up to $115 \mathrm{mT}$ could obtain. The exposure was carried out at room temperature. The intensity of the field was controlled through the DC current. Time of exposure was examined versus the intensity of exposure. No magnetic field shielding was used. The magnetic field of earth and background was almost zero and neglected. The effect of temperature on blood samples neglected, temperature increase measured during calibration was a $2^{\circ}$

TABLE V

Blood Counts Under 75 Mt of Static Magnetic Field Versus Exposure Period. Not Significant $(P>0.05)$

\begin{tabular}{|c|c|c|c|c|c|}
\hline \multirow{2}{*}{$\begin{array}{l}\mathrm{B}=75 \mathrm{mT} \\
\text { Parameters/period }\end{array}$} & \multicolumn{5}{|c|}{ Exposure time } \\
\hline & Control & $15 \mathrm{~min}$ & $30 \mathrm{~min}$ & $45 \mathrm{~min}$ & $60 \mathrm{~min}$ \\
\hline WBCs $\left(10^{9} / 1\right)$ & $5.1 \pm 0.37$ & $5.5 \pm 0.33$ & $5.3 \pm 0.14$ & $5 \pm 0.12$ & $5 \pm 0.26$ \\
\hline LYM $\left(10^{9} / 1\right)$ & $3.7 \pm 0.14$ & $4.1 \pm 0.24$ & $3.9 \pm 0.05$ & $3.8 \pm 0.24$ & $3.6 \pm 0.09$ \\
\hline LYM\% & $71.3 \pm 0.80$ & $73.5 \pm 1.18$ & $72.2 \pm 0.38$ & $73.7 \pm 0.14$ & $72.2 \pm 0.38$ \\
\hline MID $\left(10^{9} / 1\right)$ & $0.2 \pm 0.00$ & $0.3 \pm 0.02$ & $0.3 \pm 0.09$ & $0.2 \pm 0.02$ & $0.3 \pm 0.09$ \\
\hline MID \% & $5.1 \pm 0.28$ & $6 \pm 0.24$ & $5.7 \pm 0.19$ & $5.3 \pm 0.33$ & $5.7 \pm 0.19$ \\
\hline $\operatorname{RBCs}\left(10^{12} / 1\right)$ & $5.78 \pm 0.10$ & $5.79 \pm 0.10$ & $5.9 \pm 0.28$ & $5.86 \pm 0.07$ & $5.9 \pm 0.28$ \\
\hline $\mathrm{HGB}(\mathrm{g} / \mathrm{dl})$ & $12.6 \pm 0.19$ & $12.9 \pm 0.24$ & $13.1 \pm 0.52$ & $12.9 \pm 0.57$ & $13.1 \pm 0.52$ \\
\hline НCT\% & $36.6 \pm 0.66$ & $36.1 \pm 0.71$ & $36.8 \pm 0.33$ & $36.5 \pm 5.12$ & $36.8 \pm 0.33$ \\
\hline MCV (fl) & $63.4 \pm 1.70$ & $62.4 \pm 0.52$ & $62.4 \pm 0.14$ & $62.3 \pm 3.12$ & $62.4 \pm 0.14$ \\
\hline $\mathrm{MCH}(\mathrm{pg})$ & $21.8 \pm 0.66$ & $22.2 \pm 0.85$ & $22.1 \pm 1.04$ & $22 \pm 0.12$ & $22.1 \pm 1.04$ \\
\hline $\mathrm{MCHC}(\mathrm{g} / \mathrm{dl})$ & $34.4 \pm 0.66$ & $35.6 \pm 0.66$ & $35.5 \pm 0.38$ & $35.3 \pm 3.12$ & $35.5 \pm 0.38$ \\
\hline PDWa (fl) & $9.2 \pm 0.52$ & $9 \pm 0.61$ & $9 \pm 0.28$ & $9 \pm 0.12$ & $9 \pm 0.28$ \\
\hline PDW\% & $38.3 \pm 0.82$ & $37.9 \pm 0.75$ & $37.9 \pm 0.05$ & $37.8 \pm 8.12$ & $37.9 \pm 0.05$ \\
\hline РCT\% & $0.45 \pm 0.09$ & $0.43 \pm 0.08$ & $0.42 \pm 0.02$ & $0.41 \pm 0.02$ & $0.42 \pm 0.02$ \\
\hline P-LCR\% & $4.9 \pm 0.24$ & $5 \pm 0.28$ & $3.8 \pm 0.09$ & $4 \pm 0.12$ & $3.8 \pm 0.09$ \\
\hline P-LCC $\left(10^{9} / 1\right)$ & $35 \pm 0.94$ & $35 \pm 0.94$ & $26 \pm 0.80$ & $26 \pm 0.12$ & $26 \pm 0.80$ \\
\hline
\end{tabular}

WBCs: White blood cells, RBCs: Red blood cells, HGB: Hemoglobin, PLT: Platelet

TABLE VI

Blood Counts Under 100 Mt of Static Magnetic Field Versus Exposure Period. Not Significant $(P>0.05)$

\begin{tabular}{|c|c|c|c|c|c|}
\hline \multirow{2}{*}{$\begin{array}{l}\mathrm{B}=100 \mathrm{mT} \\
\text { Parameters/period }\end{array}$} & \multicolumn{5}{|c|}{ Exposure time } \\
\hline & Control & $15 \mathrm{~min}$ & $30 \mathrm{~min}$ & $45 \mathrm{~min}$ & $60 \mathrm{~min}$ \\
\hline WBCs $\left(10^{9} / 1\right)$ & $5 \pm 0.50$ & $5.1 \pm 0.54$ & $5.2 \pm 0.12$ & $5.3 \pm 0.83$ & $4.9 \pm 0.62$ \\
\hline LYM $\left(10^{9} / 1\right)$ & $4 \pm 0.14$ & $4.3 \pm 0.24$ & $4.1 \pm 0.14$ & $4.3 \pm 0.33$ & $4 \pm 0.47$ \\
\hline $\operatorname{MID}\left(10^{9} / 1\right)$ & $0.2 \pm 0.00$ & $0.5 \pm 0.05$ & $0.2 \pm 0.00$ & $0.2 \pm 0.02$ & $0.2 \pm 0.03$ \\
\hline MID \% & $4.3 \pm 0.33$ & $9.7 \pm 0.33$ & $4.9 \pm 0.14$ & $4.3 \pm 0.33$ & $4.4 \pm 0.28$ \\
\hline GRA $\left(10^{9} / 1\right)$ & $0.8 \pm 0.09$ & $0.3 \pm 0.02$ & $0.9 \pm 0.19$ & $0.8 \pm 0.09$ & $0.7 \pm 0.14$ \\
\hline $\operatorname{RBCs}\left(10^{12} / 1\right)$ & $6.45 \pm 0.45$ & $6.2 \pm 0.42$ & $6.48 \pm 0.48$ & $6.47 \pm 0.25$ & $6.44 \pm 0.2$ \\
\hline HGB (g/dl) & $13.5 \pm 0.47$ & $13.3 \pm 0.61$ & $14 \pm 0.61$ & $14 \pm 0.94$ & $13.7 \pm 0.8$ \\
\hline НCТ\% & $40.4 \pm 0.90$ & $38.6 \pm 0.05$ & $40 \pm 1.13$ & $40 \pm 0.94$ & $40.1 \pm 0.9$ \\
\hline MCV (fl) & $62.6 \pm 0.80$ & $62.2 \pm 0.47$ & $61.7 \pm 0.38$ & $61.9 \pm 0.05$ & $62.2 \pm 0.8$ \\
\hline $\mathrm{MCH}(\mathrm{pg})$ & $20.9 \pm 0.75$ & $21.5 \pm 0.42$ & $21.5 \pm 0.42$ & $21.7 \pm 1.08$ & $21.3 \pm 0.8$ \\
\hline $\mathrm{MCHC}(\mathrm{g} / \mathrm{dl})$ & $33.3 \pm 0.99$ & $34.6 \pm 0.85$ & $34.9 \pm 0.75$ & $35.1 \pm 0.90$ & $34.2 \pm 0.3$ \\
\hline PDWa (fl) & $9.3 \pm 0.52$ & $9.2 \pm 0.33$ & $9 \pm 0.42$ & $8.9 \pm 0.42$ & $9 \pm 0.94$ \\
\hline PDW\% & $39 \pm 0.94$ & $39 \pm 0.94$ & $38.1 \pm 0.44$ & $38.1 \pm 0.99$ & $38.6 \pm 0.1$ \\
\hline РCT\% & $0.46 \pm 0.02$ & $0.53 \pm 0.03$ & $0.40 \pm 0.02$ & $0.41 \pm 0.00$ & $0.42 \pm 0.2$ \\
\hline P-LCR\% & $6.2 \pm 0.33$ & $5.4 \pm 0.28$ & $4.7 \pm 0.33$ & $4 \pm 0.47$ & $4.2 \pm 0.38$ \\
\hline P-LCC $\left(10^{9} / 1\right)$ & $44 \pm 0.47$ & $46 \pm 1.41$ & $31 \pm 0.94$ & $27 \pm 0.94$ & $29 \pm 1.89$ \\
\hline
\end{tabular}

WBCs: White blood cells, RBCs: Red blood cells, HGB: Hemoglobin, PLT: Platelet 
increase after $1 \mathrm{~h}$ operating the exposure set-up. Both control and exposure sample left at the same room and atmosphere.

\section{Hematological Analysis}

Medonic M-series M32 hematology analyzers (made in Sweden) were used to examine blood counts. It performs the test ultrafast with fully differential parameters. Student's $t$-test was used to extract significance of changes. Microsoft Office 2019 program employed to elicit the rest of data analysis and charts configuration. Percentage of changes calculated in compare to control samples.

\section{RESULTS}

Tables I-VI represent the blood count changes under the effect of magnetic field $(2.4,6,25,50,75$, and $100 \mathrm{mT}$, respectively) versus time of exposure (15, 30, 45, and $60 \mathrm{~min})$. Blood count alterations plotted after exposure in ratio to control values (before exposure) are shown in Figs. 2-5. Fig. 2 represents the alteration in main blood parameters. Overall exposure, a $2.4 \mathrm{mT}$ SMF resulted a significant $(P<0.05)$ impact on WBCs and PLTs, yet, insignificantly on RBCs. For instance, a 15 min exposure raised WBCs with $31 \%$ and reduced PLTs with $29 \%$. However, this figure has changed at 60 min exposure, whereas WBCs count increased and PLTs count reduced with $18 \%$ and $35 \%$, respectively. RBCs fluctuated from $2 \%$ to $5 \%$ as blood samples exposed from 15 to $60 \mathrm{~min}$.

Blood samples exposed to a $6 \mathrm{mT}$ : $45 \mathrm{~min}$ displayed a tremendous reduction of WBCs and PLTs, $40 \%$ and $51 \%$, respectively. The 15-60 min of exposure reduced WBCs count from $3 \%$ to $40 \%$ non-linearly. RBCs count increase
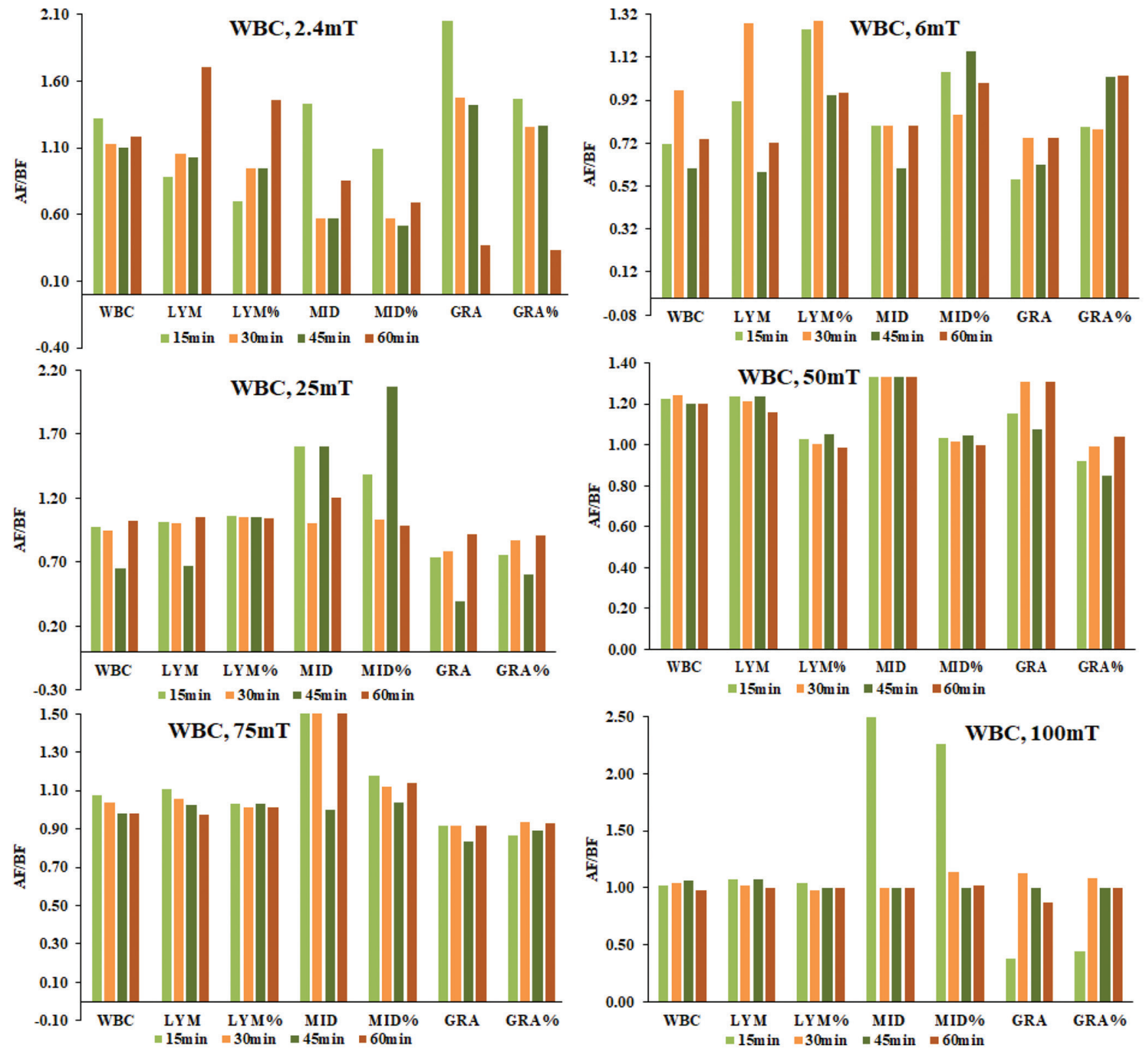

Fig. 3. White blood cell parameters change under the effect of various static magnetic field intensity and exposure duration. Y-axis represents the ratio between results after exposure to before exposure. A value above "1" represents an increase and below "1" is a decrease compared to control results. 
fluctuated from $1 \%$ to $6 \%$. A remarkable reduces of $\mathrm{WBCs}$, PLTs, and RBCs count obtained with $35 \%, 28 \%$, and $13 \%$, respectively, under the $25 \mathrm{mT}$ : $45 \mathrm{~min}$. The lower and longer exposure time resulted changes as small as $2 \%$.

The $50 \mathrm{mT}$ of exposure increased WBCs significantly $(P<005)$, about $20 \%$ versus exposure period, compare to an insignificant increase in RBCs and PLTs values, RBCs: $3-6 \%$ and PLTs: $1-4 \%$. The $75 \mathrm{mT}$ exposure did not enhance blood parameters significantly $(P>0.05)$. The $15 \mathrm{~min}$ of exposure increased the WBCs levels with only $7 \%$. Other variations were as small as $1 \%$. The highest exposure field $(100 \mathrm{mT})$ showed a similar response except an enhancement in PLTs level with $17 \%$ obtained due to $15 \mathrm{~min}$ of exposure. Overall result reveals a non-significant response of WBCs with 75 $\mathrm{mT}$ and $100 \mathrm{mT}$. Concerning RBCs counts, 13\% reduction was the highest obtained at $25 \mathrm{mT}, 45 \mathrm{~min}$. Therefore, none of other exposure field/duration is $6 \%$ exceeded. In contrast, WBCs level exceeded 39\%. PLTs count dropped dramatically as exposure field increased.

There was an increase trend of HGB, HTC content, and lymphocytes $(16 \%, 21 \%$, and $71 \%)$ in low exposure field $(2.4$ $\mathrm{mT}, 60 \mathrm{~min}$ ) but not at a higher exposure field. In addition, granulocytes have increased tremendously (doubled: 2.4 $\mathrm{mT}, 15 \mathrm{~min}$ ) as well as $63 \%$ reduction at $60 \mathrm{~min}$ exposure. The HTC percentage caused no alteration at high exposure field. The sharp change was $22 \%$ increase at $2.4 \mathrm{mT}, 60 \mathrm{~min}$ and $13 \%$ reduction at $25 \mathrm{mT}, 15 \mathrm{~min}$. The exposure field did not produce any impact on platelet distribution width. Nevertheless, $11 \%$ increase of RBC distribution width reported at low field exposure, $2.4 \mathrm{mT}$ : $15 \mathrm{~min}$.
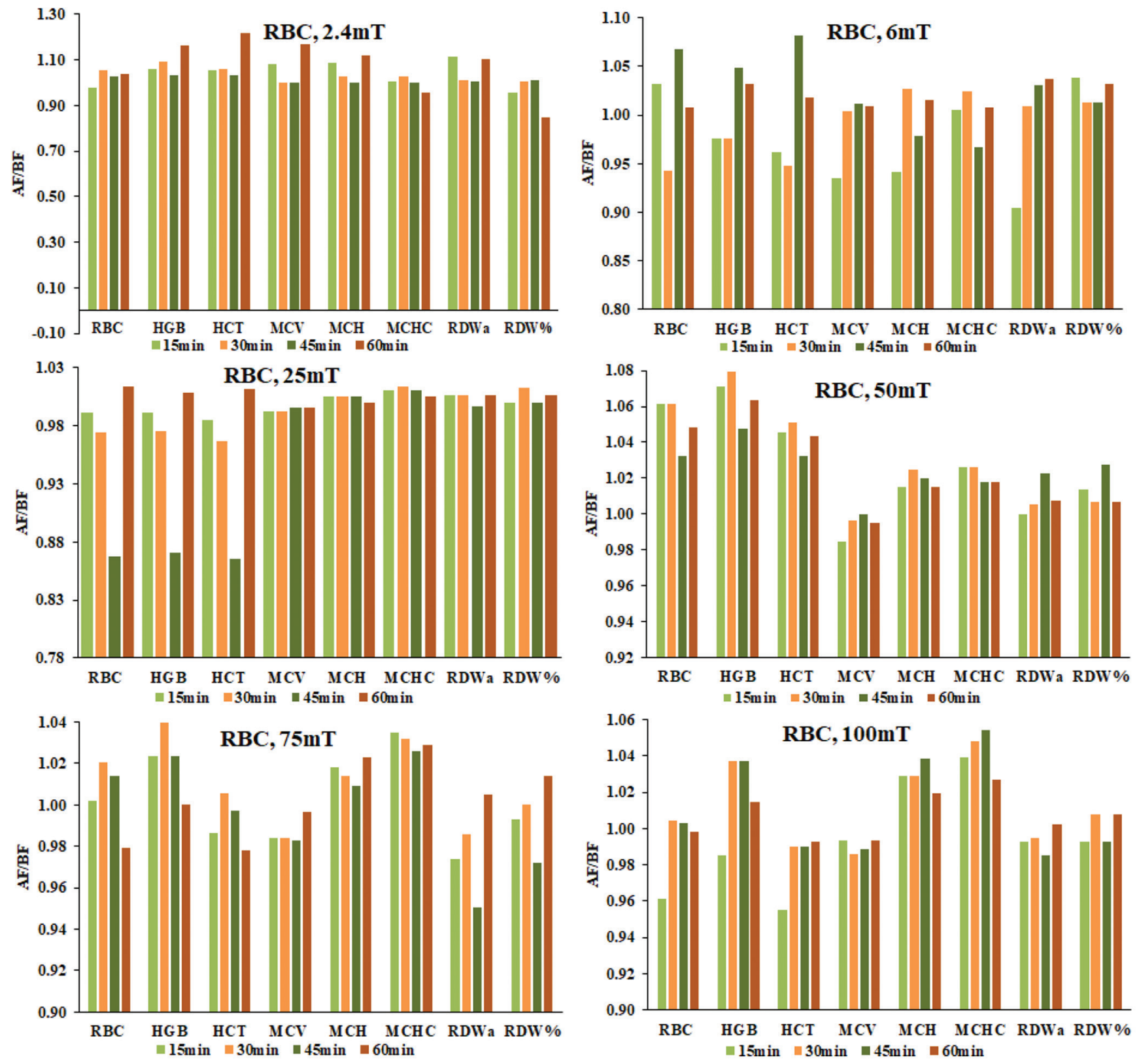

Fig. 4. Red blood cells count variation under the effect of magnetic field intensity versus exposure time. Y-axis represents the ratio between results after exposure to before exposure. A value above " 1 " represents an increase and below " 1 " is a decrease compared to control results. 


\section{DisCUSSION}

Our study revealed that low exposure SMF enhances WBCs and PLTs count significantly $(P<005)$ inconsistent to a less significant increase of RBCs HGB and HTC levels. Similar trend obtained by Chater, et al., 2006, however, their results are inconsistent to a significant RBCs and WBCs variation. HGB counts are RBCs variation correlated. In addition, finding RBCs concentration far from normal can be related to anima diseases (Blann, 2014). It is believed that changes of RBCs count are mainly aggregation related. A decrease of RBCs aggregation leads to an increase of RBCs count. SMF enhanced aggregation examined by Mustafa, et al., 2020b. According to their investigation, 42.5 mT SMF decreased RBCs count significantly after $30 \mathrm{~min}$ exposure in vitro experiment, a similar result obtained in our study at low exposure field. $\mathrm{HGB}$ and $\mathrm{HTC} \%$ enhanced due to $34.8 \mathrm{mT}$ and $15 \mathrm{~min}$.

The previous studies revealed pro-inflammatory changes and reactive oxygen species production under the influence of SMF (Sahebjamei, et al., 2007; Zhao, et al., 2011). It shows that HGB is sensitive to SMF. An increase of HGB and HTC levels was summarized as hypoxia-like status (Sihem, et al., 2006). This can be the result of oxygenbinding impairment. RBCs orientation and alignment were demonstrated under magnetic field (Yamagishi, 1990). RBCs orientation increases blood viscosity (Strieth, et al., 2008). Increasing hematological parameters may cause significant cardiovascular risk including vascular reactivity, heart stroke, and blood disorder. The ventricular function is impaired when the HGB level reaches into its $50 \%$ reduction. In addition,
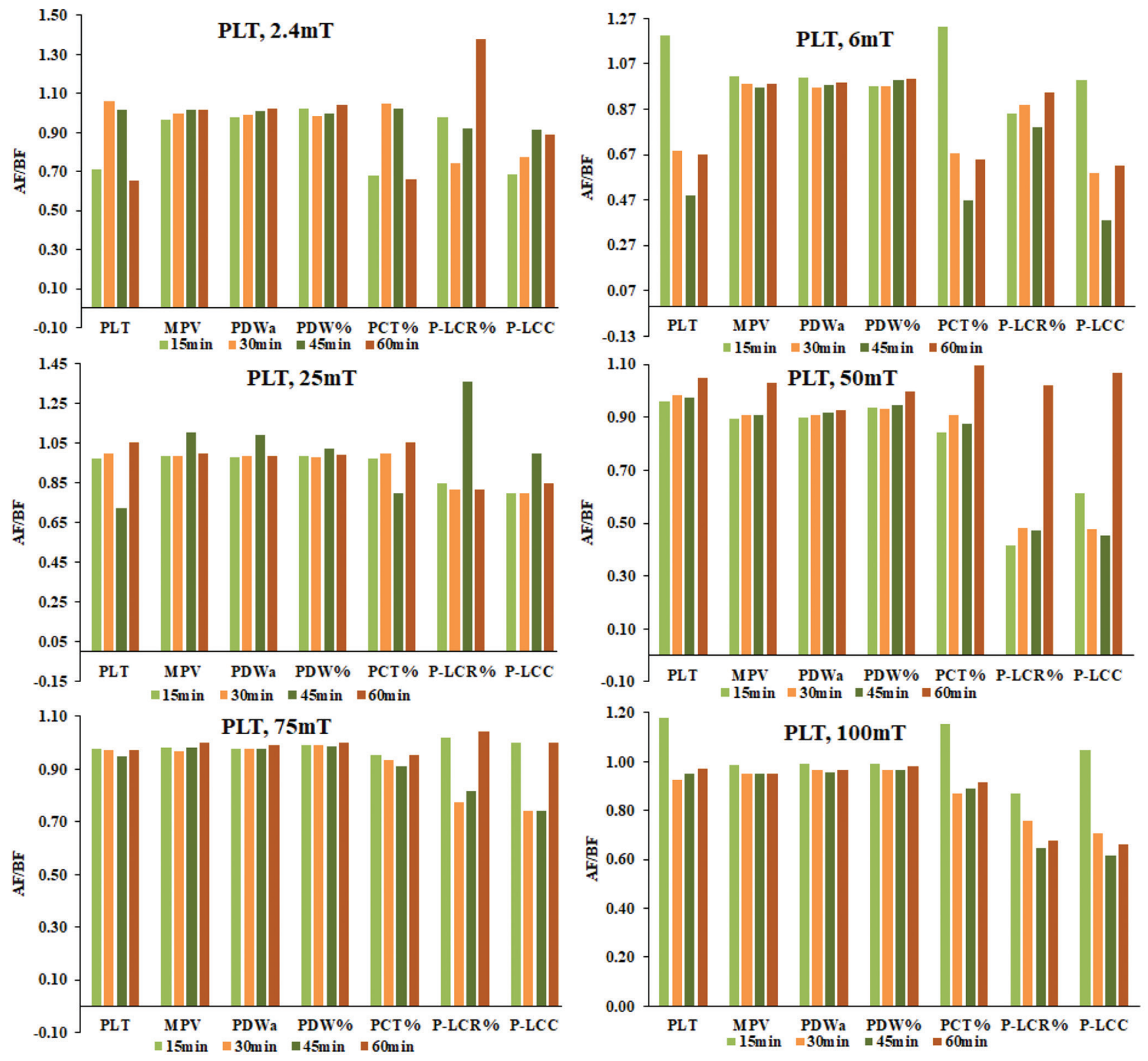

Fig. 5. Blood platelets count variation under the effect of intensity change of magnetic field versus exposure period. Y-axis represents the ratio between results after exposure to before exposure. A value above "1" represents an increase and below "1" is a decrease compared to control results. 
the reduction of HGB concentration causes inadequate oxygen support to body tissues, ventricular remodeling, and raising peripheral resistance. These factors contribute the risk of ischemia or its related symptoms (Maulood, 2018).

WBCs (leukocytes) are immunity cells beneficial to fight infections and diseases including AIDS, leukemia, and certain types of blood cancers (AL-Dulaimi, et al., 2018). Leukocyte counts linkage with coronary cardiac diseases was found to produce an independent risk factor. For example, the risk of death related to a $65 \%$ increase of leukocyte counts was shown from ischemic heart diseases (Sweetnam, et al., 1997). A small increase of leukocyte counts under the effect of magnetic field contributed an increase of spleen cellularity. In our experiment, granulocyte counts increased significantly under the effect of $2.4,6$, and $25 \mathrm{mT}$. This result is similar with $16 \mathrm{mT}$ exposure mice where the granulocyte counts raised comparable, regardless to unchanged of RBC counts after 30 days exposure (Djordjevich, et al., 2012). PLTs are a nucleate cellular fragments and essential hemostasis carriers (Italiano, 2007). Disorder in PLTs causes bleeding disorder, which can progress to viral hemorrhagic fevers and can progress to shock and death (Zapata, et al., 2014). With the above discussion, blood cells count variation showed in this study under SMF required extra attention.

\section{Conclusion}

Influence of a homogenous and uniform SMF on the main blood cell counts in vitro experiment for the albino investigated. It has concluded that the significant changes of blood cell counts under the low field effects increased non-linearly, and the high exposure SMF did not enhanced blood parameters. WBCs demonstrated the maximal response compering with the RBC and PLT. RBC indices have not influenced with SMF. The overall change of PLTs was a decrease. From this study, it can be concluded that low static field can interact with WBCs and blood PLTs significantly.

\section{ACKNOWLEDGMENT}

The authors give thanks and appreciation to the operational and academic staffs in the blood analysis and medical physics laboratories for their support and facility.

\section{CONFLICTS OF INTEREST}

The authors declared that they have no conflicts of interest.

\section{REFERENCES}

Aida, L., Soumaya, G., Myriam, E., Mohsen, S. and Hafedh, A., 2014. Effects of static magnetic field exposure on plasma element levels in rat. Biological Trace Element Research, 160, pp.67-72.

AL-Dulaimi, K., Banks, J., Chandran, V., Tomeo-Reyes, I. and Nguyen, K., 2018. Classification of white blood cell types from microscope images: Techniques and challenges. In: Microscopy Science: Last Approaches on Educational Programs and Applied Research. Formatex Research Center, Spain, pp.17-25.
Blann, A., 2014. Functions and diseases of red and white blood cells. Nurs Times, 110, pp.16-18.

Chadwick, P. and Lowesf, F., 1998. Magnetic fields on british trains. Annals Occupational Hygiene, 42, pp.331-335.

Chater, S., Abdelmelek, H., Pequignot, J.M., Sakly, M. and Ben Rhouma, K., 2006. Effects of sub-acute exposure to static magnetic field on hematologic and biochemical parameters in pregnant rats. Electromagnetic Biology Medicine, 25, pp.135-144.

Djordjevich, D.M., Luka, S.R. De, Milovanovich, I.D., Andjelija, Z., Stefanovic, S., Trbovich, A.M. and Ristic, J.L., 2012. Ecotoxicology and environmental safety hematological parameters' changes in mice subchronically exposed to static magnetic fields of different orientations. Ecotoxicology Environmental Safety, 81, pp.8-105.

Hashish, A.H., El-Missiry, M.A., Abdelkader, H.I. and Abou-Saleh, R.H., 2008. Assessment of biological changes of continuous whole body exposure to static magnetic field and extremely low frequency electromagnetic fields in mice. Ecotoxicology Environmental Safety, 71, pp.895-902.

Ismail, A.H., 2015. Influence of radiation doses on the ratio of blast cells, lymphocytes and neutrophils inside blood samples of leukaemia patients: In vitro. ZANCO Journal of Pure and Applied Sciences, 27(3), pp.65-74.

Italiano, J.E., 2007. The structure and production of blood platelets. In: Platelets in Hematologic and Cardiovascular Disorders. Cambridge University Press, Cambridge, United Kingdom, pp.1-20.

Najam, L., AL-Dulamey, Q., and Al-Jawwady, Y., 2020. Effects of low dose gamma ray on some hematological parameters in adult rats. Iranian Journal of Medical Physics, 17(3), pp.137-141.

Maulood, K.A., 2018. Assessment of some hematological, biochemical parameters and cardiac biomarker levels in patients with ischemic heart disease in Erbil city. Zanco Journal of Pure Applied Sciences, 30, pp.86-93.

Milovanovich, I.D., Luka, S.R. De, Djordjevich, D.M., Ili, A.Ž., Popovi, T., Arsi, A., Obradovi, D.D., Opri, D., Risti, J.L. and Trbovich, A.M., 2016. Homogeneous static magnetic field of different orientation induces biological changes in subacutely exposed mice. Environmental Science and Pollution Research International, 23, pp.1584-1597.

Mustafa, B.T., Yab, S.P. and Ismail, A.H., 2020a. Impacts of materials on the intensity of uniform static magnetic fields using a multi Helmholtz coils design. AIP Conference Proceedings, 2213(1), pp.020063.

Mustafa, B.T., Yaba, S.P. and Ismail, A.H., 2019. A review of the effects of magnetic field on main blood cells: In vivo and in vitro experiments. ZANCO Journal of Pure and Applied Sciences, 31(6), pp.40-50.

Mustafa, B.T., Yaba, S.P. and Ismail, A.H., 2020b. Influence of static magnetic field on red blood cells parameters and platelets using tests of $\mathrm{CBC}$ and microscopy images. Biomedical Physics and Engineering Express, 6(2), pp.025004.

Okano, H. and Ohkubo, C., 2005. Effects of neck exposure to $5.5 \mathrm{mT}$ static magnetic field on pharmacologically modulated blood pressure in conscious rabbits. Bioelectromagnetics, 26, pp.469-480.

Sahebjamei, H., Abdolmaleki, P. and Ghanati, F., 2007. Effects of magnetic field on the antioxidant enzyme activities of suspension-cultured tobacco cells. Bioelectromagnetics, 28, pp.42-47.

Sihem, C., Hafedh, A., Mohsen, S., Marc, P.J. and Khmais, B.R., 2006. Effects of sub-acute exposure to magnetic field on blood hematological and biochemical parameters in female rats. Turkish Journal of Hematology, 23(4), pp.182-187.

Strieth, S., Strelczyk, D., Eichhorn, M.E., Dellian, M., Luedemann, S., Griebel, J., Bellemann, M., Berghaus, A. and Brix, G., 2008. Static magnetic fields induce blood flow decrease and platelet adherence in tumor microvessels. Cancer Biology and Therapy, 7, pp.632-637.

Sweetnam, P.M., Thomas, H.F., Yarnell, J.W.G., Baker, I.A. and Elwood, P.C., 1997. Total and differential leukocyte counts as predictors of ischemic 
heart disease: The caerphilly and speedwell studies. American Journal of Epidemiology, 145, pp.416-421.

Van Deventer, T.E., Saunders, R. and Repacholi, M.H., 2005. WHO health risk assessment process for static fields. Progress in Biophysics and Molecular Biology, 87, pp.355-363.

Vergallo, C. and Dini, L., 2018. Comparative analysis of biological effects induced on different cell types by magnetic fields with magnetic flux densities in the range of 1-60 mT and frequencies up to $50 \mathrm{~Hz}$. Sustainability, 10(8), pp.2776.

World Health Organization, 2006. Static fields, Environmental Health Criteria. Geneva: World Health Organization.
Yamagishi, A., 1990. Biological systems in high magnetic field. Journal of Magnetism and Magnetic Materials, 90-91, pp.43-46.

Zapata, J.C., Cox, D. and Salvato, M.S., 2014. The role of platelets in the pathogenesis of viral hemorrhagic fevers. PLoS Neglected Tropical Diseases, 8, pp.e2858.

Zhao, G., Chen, S., Wang, L., Zhao, Y., Wang, J., Wang, X., Zhang, W.W., Wu, R., Wu, L., Wu, Y. and Xu, A., 2011. Cellular ATP content was decreased by a homogeneous $8.5 \mathrm{~T}$ static magnetic field exposures Role of reactive oxygen species. Bioelectromagnetics, 32, pp.94-101. 